\title{
Research Article \\ Soft Separation Axioms and Fixed Soft Points Using Soft Semiopen Sets
}

\author{
T. M. Al-shami \\ Department of Mathematics, Sana'a University, Sana'a, Yemen \\ Correspondence should be addressed to T. M. Al-shami; tareqalshami83@gmail.com
}

Received 1 August 2020; Revised 28 October 2020; Accepted 30 October 2020; Published 16 November 2020

Academic Editor: Bruno Carpentieri

Copyright (c) 2020 T. M. Al-shami. This is an open access article distributed under the Creative Commons Attribution License, which permits unrestricted use, distribution, and reproduction in any medium, provided the original work is properly cited.

\begin{abstract}
The importance of soft separation axioms comes from their vital role in classifications of soft spaces, and their interesting properties are studied. This article is devoted to introducing the concepts of $t$-soft semi- $T_{i}(i=0,1,2,3,4)$ and $t t$-soft semiregular spaces with respect to ordinary points. We formulate them by utilizing the relations of total belong and total nonbelong. The advantages behind using these relations are, first, generalization of existing comparable properties on general topology and, second, eliminating the stability shape of soft open and closed subsets of soft semiregular spaces. By some examples, we show the relationships between them as well as with soft semi- $T_{i}(i=0,1,2,3,4)$ and soft semiregular spaces. Also, we explore under what conditions they are kept between soft topology and its parametric topologies. We characterize a $t$-soft semiregular space and demonstrate that it guarantees the equivalence of $t t$-soft $\operatorname{semi}-T_{i}(i=0,1,2)$. Further, we investigate some interrelations of them and some soft topological notions such as soft compactness, product soft spaces, and sum of soft topological spaces. Finally, we define a concept of semifixed soft point and study its main properties.
\end{abstract}

\section{Introduction}

In daily life, human beings face different kinds of uncertainties in fields such as economics, environmental and social sciences, engineering, medicine etc. To tackle such uncertainties, different approaches were proposed like fuzzy and rough set theories. However, they have their own limitations. Molodtsov [1] introduced an effective tool that is free from these limitations to solve uncertainties, namely soft set. This tool does not require specific type of parameters. Instead, it accommodates all types of parameters such as words, numbers, sentences, and functions. After that, Maji et al. [2] established some fundamental operations between two soft sets and then tackled one of their applications in decisionmaking problems [3]. But the authors of [4] demonstrated some shortcomings of the operations defined in [2]. They also explored novel operations between soft sets which became a goal of many studies on algebraic soft structures. Al-shami and El-Shafei [5] defined new types of soft subset and equality relations and then applied them on soft linear equations.
In 2011, Shabir and Naz [6] defined a soft topological space over a family of soft sets. They constructed the fundamental notions of soft topological spaces such as the operators of soft closure and interior, soft subspace, and soft separation axioms. Min [7] continued studying soft separation axioms and corrected some alleged results in [6]. Then, Aygünoglu and Aygün [8] scrutinized the concept of compactness on soft setting. By exploiting one of the diverges between soft sets and crisp sets, Hida [9] studied a strong type of soft compactness. Al-shami $[10,11]$ revised many results given in many studies of soft separation axioms. Then, Al-shami et al. [12] introduced a weak type of soft compactness, namely almost soft compactness. The authors of [13] presented soft maps by using two crisp maps, one of them between the sets of parameters and the second one between the universal sets. However, the authors of [14] introduced soft maps by using the concept of soft points. Some applications of different types of soft maps were the goal of some articles (see [13-15]).

Until 2018, the belong and nonbelong relations that utilized in these studies are those given by [6]. In 2018, the 
authors of [16] came up new relations of belong and nonbelong between an ordinary points and soft set, namely partial belong and total nonbelong relations. In fact, these relations widely open the door to study and redefine many soft topological notions. This leads to obtain many fruitful properties and changes which can be seen significantly on the study of soft separation axioms as it was shown in [16-18].

Das and Samanta [19] studied the concept of a soft metric based on the soft real set and soft real numbers given in [20]. Wardowski [14] tackled the fixed point in the setup of soft topological spaces. Abbas et al. [21] presented soft contraction mappings and established a soft Banach fixed point theorem in the framework of soft metric spaces. Recently, many researchers explored fixed point findings in soft metric type spaces (see, for example, [22-25]). Some results related to intuitionistic fuzzy soft sets were investigated in $[26,27]$. Recently, Alcantud [28] has discussed the properties of topological separability on soft setting.

One of the significant ideas that helps to prove some properties and removes some problems on soft topology is the concept of a soft point. It was first defined by Zorlutuna and Çakir [29] in order to study the interior points of a soft set and soft neighborhood systems. Then, [19, 30] simultaneously redefined soft points to discuss soft metric spaces. In fact, the recent definition of a soft point makes similarity between many set-theoretic properties and their counterparts on soft setting. Two types of soft topologies, namely enriched soft topology and extended soft topology, were studied in $[8,30]$, respectively. Al-shami and Kocinac [31] discussed the equivalence between these two topologies and obtained interesting results. Recently, they [32] have introduced the concept of nearly soft Menger spaces and investigated main characteristics.

We organized this paper as follows: After this introduction, Section 2 addresses the basic principles about the soft sets and soft topologies. In Section (, we introduce the concepts of $t t$-soft semi- $T_{i}(i=0,1,2,3,4)$ and $t t$-soft semiregular spaces with respect to ordinary points by using the relations of total belong and total nonbelong. The relationships between them and their main properties are discussed with the help of interesting examples. In Section 4, we explore a semifixed soft point theorem and study some main properties. In particular, we conclude under what conditions semifixed soft points are preserved between a soft topology and its parametric topologies. Finally, conclusions of the paper in Section 5 .

\section{Preliminaries}

To well understand the results obtained in this study, we shall mind some essential concepts, definitions, and properties from the literature.

\subsection{Soft Sets}

Definition 1. [1] A map $G$ of $P$ into the power set $2^{X}$ of a nonempty set $X$ is called a soft set over $X$, where $X$ is the universal set and $P$ is a set of parameters.
In this study, we use a symbol $G_{P}$ to refer a soft set, and we identify it as ordered pairs $G_{P}=\{(p, G(p)): p \in P$ and $\left.G(p) \in 2^{X}\right\}$.

A class of all soft sets defined over $X$ with $P$ is symbolized by $S\left(X_{P}\right)$.

Definition 2. [33] $G_{P}$ is said to be a subset of $H_{P}$, symbolized by $G_{P} \tilde{\subseteq} H_{P}$, if $G(p) \subseteq H(p)$ for each $p \in P$.

We write $G_{P}=H_{P}$ provided that $G_{P} \tilde{\subseteq} H_{P}$ and $H_{P} \tilde{\subseteq} G_{P}$.

Definition 3. $[6,16]$ For a soft set $G_{P}$ and $w \in X$, we say that

(i) $w \in G_{P}$, it is read as follows: $w$ totally belongs to $G_{P}$, if $w \in G(p)$ for each $p \in P$

(ii) $w \notin G_{P}$, it is read as follows: $w$ does not partially belong to $G_{P}$, if $w \notin G(p)$ for some $p \in P$

(iii) $w \Subset G_{P}$, it is read as follows: $w$ partially belongs to $G_{P}$, if $w \in G(p)$ for some $p \in P$

(iv) $w \notin G_{P}$, it is read as follows: $w$ does not totally belong to $G_{P}$, if $w \notin G(p)$ for each $p \in P$.

Definition 4. [4] The relative complement of $G_{P}$ is another soft set $G_{P}^{c}$, where a map $G^{c}: P \longrightarrow 2^{X}$ is defined by $G^{c}(p)=$ $X \backslash G(p)$ for all $p \in P$.

Definition 5. $[2,16,19,30]$ A soft set $\left(G_{P}\right)$ over $X$ is said to be

(i) a null soft set (resp., an absolute soft set), symbolized by $\tilde{\Phi}$ (resp., $\tilde{X}$ ), if $G(p)$ is the empty (resp., universal) set for each $p \in P$

(ii) a soft point $S_{p}^{x}$ if there are $p \in P$ and $x \in X$ so that $G$ $(p)=\{x\}$ and $G\left(p^{\prime}\right)=\varnothing$ for each $p^{\prime} \in P \backslash\{p\}$. We write that $S_{p}^{x} \in G_{P}$ if $x \in G(p)$

(iii) a stable soft set if all p-approximate are equal $\mathrm{A}$ and it is symbolized by $\tilde{A}$. In particular, we symbolize by $x_{P}$ if $A=\{x\}$

(iv) a countable (resp., finite) soft set if $G(p)$ is countable (resp., finite) for each $p \in P$. Otherwise, it is said to be uncountable (resp., infinite).

Definition 6. $[2,4]$ The intersection and union of $G_{P}$ and $H_{P}$ are defined as follows:

(i) Their intersection, symbolized by $G_{P} \tilde{\cap} H_{P}$, is a soft set $U_{P}$, where a mapping $U: P \longrightarrow 2^{X}$ is given by $U(p)=G(p) \cap H(p)$

(ii) Their union, symbolized by $G_{P} \widetilde{\cup} H_{P}$, is a soft set $U_{P}$, where a mapping $U: P \longrightarrow 2^{X}$ is given by $U(p)=$ $G(p) \cup H(p)$. 
The union and intersection operators were generalized for any number of soft sets in a similar way.

Definition 7. [34] The Cartesian product of $G_{P}$ and $H_{F}$, symbolized by $G \times H_{P \times F}$, is defined as $(G \times H)(p, f)=$ $G(p) \times H(f)$ for each $(p, f) \in P \times F$.

Definition 8. [13] A soft mapping of $S\left(X_{A}\right)$ into $S\left(Y_{B}\right)$, symbolized by $f_{\phi}$, is defined by two crisp maps $f: X \longrightarrow Y$ and $\phi: A \longrightarrow B$ so that the image of $G_{A}$ is a set in $S\left(X_{A}\right)$ and pre-image of $H_{B}$ is a set in $S\left(Y_{B}\right)$ and they are defined as follows.

(i) $f_{\phi}\left(G_{A}\right)=\left(f_{\phi}(G)\right)_{B}$ is a set in $S\left(Y_{B}\right)$, where $f_{\phi}(G)(b)$ $=\bigcup_{a \in \phi^{-1}(b)} f(G(a))$ for each $b \in B$

(ii) $f_{\phi}^{-1}\left(H_{B}\right)=\left(f_{\phi}^{-1}(H)\right)_{A}$ is a set in $S\left(X_{A}\right)$, where $f_{\phi}^{-1}(H)(a)=f^{-1}(H(\phi(a)))$ for each $a \in A$.

Definition 9. [29] $f_{\phi}: S\left(X_{A}\right) \longrightarrow S\left(Y_{B}\right)$ is called a $\lambda$ soft map provided that $\phi$ and $f$ are $\lambda$ maps, where $\lambda \in\{$ injective, surjective, and bijective $\}$.

\subsection{Soft Topology}

Definition 10. [6] A family $\Omega$ of soft sets over $X$ under a fixed set of parameters $P$ is said to be a soft topology on $X$ if it satisfies the following:

(i) $\tilde{X}$ and $\tilde{\Phi}$ are elements of $\Omega$

(ii) The intersection of a finite number of $\Omega$ is an element of $\Omega$

(iii) The union of an arbitrary number of $\Omega$ is an element of $\Omega$.

We will call a term of soft topological space on the triple $(X, \Omega, P)$, where a terminology of soft open is given for every member in $\Omega$ and a terminology of soft closed is given for a relative complement of a member of $\Omega$.

Proposition 11. [6] In $(X, \Omega, P)$, a family $\Omega_{p}=\{G(p)$ : $\left.G_{P} \in \Omega\right\}$ is a classical topology on $X$ for each $p \in P$.

We will call a term of parametric topology on $\Omega_{p}$ and a term of parametric topological space on $\left(X, \Omega_{p}\right)$.

Definition 12. [6] $\left(Y, \Omega_{Y}, P\right)$ is called a soft subspace of $(X$, $\Omega, P)$, where $\varnothing \neq Y \subseteq X$ and $\Omega_{Y}=\left\{\tilde{Y} \cap G_{P}: G_{P} \in \Omega\right\}$.

Definition 13. [35] $G_{P}$ is called a soft semiopen subset of $(X$, $\Omega, P)$ if $G_{P} \subseteq \sim \operatorname{cl}\left(\right.$ int $\left.\left(G_{P}\right)\right)$.

Theorem 14. [35] Every soft open set is soft semiopen, and the union of an arbitrary number of soft semiopen sets is soft semiopen.
Definition 15. [35] The intersection of all soft semiclosed sets in $(X, \Omega, P)$ containing $G_{P}$ is the soft preclosure of $G_{P}$. It is symbolized by $\bar{G}_{P}{ }^{s}$.

It is clear that $x \in \bar{G}_{P}{ }^{s}$ iff for each soft semiopen set $U_{P}$ totally containing $x$ we have $G_{P} \tilde{\cap} U_{P} \neq \tilde{\Phi}$; and $S_{p}^{x} \in \bar{G}_{P}{ }^{s}$ iff for each soft semiopen set $U_{P}$ totally containing $S_{p}^{x}$ we have $G_{P} \tilde{\cap} U_{P} \neq \tilde{\Phi}$.

Definition 16. [36] $(X, \Omega, P)$ which does not contain disjoint soft open sets is called soft hyperconnected.

The following result will help us establish some properties of soft semiseparation axioms and soft semicompact spaces (see, for example, Theorem 42 and Proposition 49). It implies that the family of soft semiopen subsets of a soft hyperconnected space $(X, \Omega, P)$ forms a new soft topology $\Omega^{\text {semi }}$ over $X$ that is finer than $\Omega$.

Theorem 17. [37] A class of soft semiopen subsets of a soft hyperconnected space forms a soft topology.

Proposition 18. Let $\tilde{Y}$ be soft open set in $(X, \Omega, P)$. Then

(1) If $(H, P)$ is soft semiopen and $\tilde{Y}$ is soft open set in $(X, \Omega, P)$, then $(H, P) \tilde{\cap}(Y, P)$ is a soft semiopen set in $\left(Y, \Omega_{Y}, P\right)$

(2) If $\tilde{Y}$ is soft open in $(X, \Omega, P)$ and $(H, P)$ is a soft semiopen in $\left(Y, \Omega_{Y}, P\right)$, then $(H, P)$ is a soft semiopen set in $(X, \Omega, P)$.

Definition 19. [35] (X, $\Omega, P)$ is said to be

(i) soft semi- $T_{0}$ if there is a soft semiopen set $U_{P}$ for each $x \neq y \in X$ satisfying $x \in U_{P}$ and $y \notin U_{P}$; or $y \in$ $U_{P}$ and $x \notin U_{P}$

(ii) soft semi- $T_{1}$ if there are two soft semiopen sets $U_{P}$ and $V_{P}$ for each $x \neq y \in X$ satisfying $x \in U_{P}$ and $y \notin$ $U_{P}$; and $y \in V_{P}$ and $x \notin V_{P}$

(iii) soft semi- $T_{2}$ if there are two disjoint soft semiopen sets $U_{P}$ and $V_{P}$ for each $x \neq y \in X$ satisfying $x \in U_{P}$ and $y \in V_{P}$

(iv) soft semiregular if every soft semiclosed set $H_{P}$ so that $x \notin H_{P}, \Omega$ contains two disjoint soft semiopen sets $U_{P}$ and $V_{P}$ satisfying $H_{P} \tilde{\simeq} U_{P}$ and $x \in V_{P}$

(v) soft seminormal if we separate every two disjoint soft semiclosed sets by two disjoint soft semiopen sets

(vi) soft semi- $T_{3}$ (resp., soft semi- $T_{4}$ ) if it is both soft semiregular (resp., soft seminormal) and soft semi$T_{1}$-space

Definition 20. [38] A soft semiopen cover of $(X, \Omega, P)$ is a family of soft semiopen sets $\left\{G_{i_{P}}: i \in I\right\}$ so that $\tilde{X}=\tilde{U}_{i \in I} G_{i_{P}}$. 
Definition 21. $(X, \Omega, P)$ is said to be

(i) soft semi- $T_{2}^{\prime}$ [37] if for every $S_{p}^{x} \neq S_{p^{\prime}}^{y} \in \tilde{X}$, there are twodisjoint soft semiopen sets $U_{P}$ and $V_{P}$ containing $S_{p}^{x}$ and $S_{p^{\prime}}^{y}$, respectively

(ii) soft semicompact [38] if every soft semiopen cover of $\tilde{X}$ has afinite subcover.

Proposition 22. [37] A soft semicompact (resp., stable soft semicompact) set in a soft semi- $T_{2}^{\prime}$-space (resp., soft semi- $T_{2}$ -space) is soft semiclosed.

To study the properties that preserved under soft semi*-homeomorphism maps, the concept of a soft semiirresolute map will be presented in this work under the name of a soft semi*-continuous map.

Definition 23. [37] $g_{\varphi}:(X, \Omega, P) \longrightarrow(Y, \theta, P)$ is called soft semi* ${ }^{*}$ continuous if the inverse image of each soft semiopen set is soft semiopen.

Proposition 24. [38] The soft semi ${ }^{*}$ - continuous image of a soft semicompact set is soft semicompact.

The definitions of soft semicontinuous, soft semiopen, soft semiclosed, and soft semihomeomorphism maps were given in [35] in a similar way of their counterparts in classical topology.

Definition 25. A soft topology $\Omega$ on $X$ is said to be

(i) an enriched soft topology [8] if all soft sets $G_{P}$ so that $G(p)=\varnothing$ or $X$ are members of $\Omega$

(ii) an extended soft topology [30] if $\Omega=\left\{G_{P}: G(p) \epsilon\right.$ $\Omega_{p}$ for each $\left.p \in P\right\}$, where $\Omega_{p}$ is a parametric topology on $X$.

Al-shami and Kočinac [31] proved that extended and enriched soft topologies are identical and obtained some useful results that help to discover the interrelations between soft topology and its parametric topologies.

Theorem 26. [31] A subset (F,P) of an extended soft topological space $(X, \Omega, P)$ is soft semiopen if and only if each p-approximate element of $(F, P)$ is semiopen.

Proposition 27. [39] Let $\left\{\left(X_{i}, \Omega_{i}, P\right): i \in I\right\}$ be a class of mutually disjoint soft topological spaces and $X=\bigcup_{i \in I} X_{i}$. Then the class $\Omega=\left\{(G, P) \tilde{\subseteq} \tilde{X}:(G, P) \tilde{\cap} \widetilde{X}_{i}\right.$ is a soft open set in $\left(X_{i}, \Omega_{i}, P\right)$ for every $\left.i \in I\right\}$.

forms a soft topology on $X$ with $P$.
Definition 28. [39] ( $X, \Omega, P)$ given in the above proposition is said to be the sum of soft topological spaces and is symbolized by $\left(\oplus_{i \in I} X_{i}, \Omega, P\right)$.

Theorem 29. [39] A soft set $(G, P) \widetilde{\widetilde{\oplus} \widetilde{\oplus_{i \in I}} X_{i}}$ is soft semiopen (resp., soft semiclosed) in $\left(\oplus_{i \in I} X_{i}, \Omega, P\right)$ if and only if all $(G, P) \tilde{\cap} \widetilde{X_{i}}$ are soft semiopen (resp., soft semiclosed) in $\left(X_{i}, \Omega_{i}, P\right)$.

Proposition 30. [14] Let $g_{\varphi}:(X, \Omega, P) \longrightarrow(X, \Omega, P)$ be a soft map so that $\tilde{\cap}_{n \in \mathbb{N}} g_{\varphi}^{n}(\tilde{X})$ is a soft point $S_{p}^{x}$. Then, $S_{p}^{x}$ is a unique fixed point of $g_{\varphi}$.

Theorem 31. [29] Let $\Omega=\left\{G_{A} \times F_{B}: G_{A} \in \Omega\right.$ and $\left.F_{B} \in \theta\right\}$, where $(X, \Omega, A)$ and $(Y, \theta, B)$ are two soft topological spaces. $A$ class of all arbitrary union of members of $\Omega$ defines a soft topology over $X \times Y$ under a fixed set of parameters $A \times B$.

Lemma 32. Let $(G, P)$ and $(H, P)$ be two subsets of $(X, \Omega, P)$ and $(Y, \theta, P)$, respectively. Then
(i) $\operatorname{cl}(G, P) \times c l(H, P)=\operatorname{cl}((G, P) \times(H, P))$
(ii) int $(G, P) \times \operatorname{int}(H, P)=\operatorname{int}((G, P) \times(H, P))$.

\section{Semisoft Separation Axioms}

In this section, the concepts of $t t$-soft $\operatorname{semi}-T_{i}(i=0,1,2,3,4)$ and $t t$-soft semiregular spaces are introduced, where $t t$ denotes the total belong and total nonbelong relations that are utilized in the definitions of these concepts. The relationships between them are shown, and their main features are studied. In addition, their behaviours with the concepts of hereditary, topological, and additive properties are investigated. Some examples are provided to elucidate the obtained results.

First of all, we see that it is necessary to classify containment into several categories as it is shown in remark below. Factually, this classification will play a vital role in redefining many soft theoretic-set and soft topological concepts, in particular, the concepts of soft interior and closure operators, soft compactness, and soft separation axioms.

Remark 33. For a soft set $G_{P}$ and $x \in X$, we have the following notes:

(i) $G_{P}$ totally contains $x$ if $x \in G_{P}$

(ii) $G_{P}$ does not partially contain $x$ if $x \notin G_{P}$

(iii) $G_{P}$ partially contains $x$ if $x \Subset G_{P}$

(iv) $G_{P}$ does not totally contain $x$ if $x \notin G_{P}$.

Definition 34. $(X, \Omega, P)$ is said to be

(i) $t t$-soft semi- $T_{0}$ if there exists a soft semiopen set $U_{P}$ for every $x \neq y \in X$ satisfying $x \in U_{P}$ and $y \notin U_{P}$ or $y \in U_{P}$ and $x \notin U_{P}$ 
(ii) $t t$-soft semi- $T_{1}$ if there exist soft semiopen sets $U_{P}$ and $V_{P}$ for every $x \neq y \in X$ satisfying $x \in U_{P}$ and $y \notin U_{P} ;$ and $y \in V_{P}$ and $x \notin V_{P}$

(iii) $t t$-soft semi- $T_{2}$ if there exist two disjoint soft semiopen sets $U_{P}$ and $V_{P}$ for every $x \neq y \in X$ satisfying $x \in U_{P}$ and $y \notin U_{P}$; and $y \in V_{P}$ and $x \notin V_{P}$

(iv) $t t$-soft semiregular if for every soft semiclosed set $H_{P}$ so that $x \notin H_{P}$, there exist disjoint soft semiopen sets $U_{P}$ and $V_{P}$ so that $H_{P} \tilde{\subseteq} U_{P}$ and $x \in V_{P}$

(v) $t t$-soft semi- $T_{3}$ (resp., $t t$-soft semi- $T_{4}$ ) if it is both $t t$-soft semiregular (resp., soft seminormal) and $t t$-soft semi- $T_{1}$.

Remark 35. It can be noted that if $F_{P}$ and $G_{P}$ are disjoint soft set, then $x \in F_{P}$ iff $x \notin G_{P}$. This implies that $(X, \Omega, P)$ is a tt-soft semi- $T_{2}$-space iff is a soft semi- $T_{2}$-space. That is, the concepts of a $t$-soft semi- $T_{2}$-space and a soft semi- $T_{2}$-space are equivalent.

We can say that $(X, \Omega, P)$ is $t t$-soft semi- $T_{2}$ if there exist two disjoint soft semiopen sets $U_{P}$ and $V_{P}$ for every $x \neq y \in X$ so that $U_{P}$ and $V_{P}$ totally contain $x$ and $y$, respectively.

Remark 36. The soft semiregular spaces imply a strict condition on the shape of soft semiopen and soft semiclosed subsets. To explain this matter, let $F_{P}$ be a soft semiclosed set so that $x \notin H_{P}$. Then, we have two cases:

(i) There are $p, p^{\prime} \in P$ so that $x \notin H(p)$ and $x \in H\left(p^{\prime}\right)$. This case is impossible because there do not exist two disjoint soft sets $U_{P}$ and $V_{P}$ containing $x$ and $H_{P}$, respectively

(ii) For each $p \in P, x \notin H(p)$. This implies that $H_{P}$ must be stable.

As a direct consequence, we infer that every soft semiclosed and soft semiopen subsets of a soft semiregular space must be stable. However, this matter does not hold on the $t t$-soft semiregular spaces because we replace a partial nonbelong relation by a total nonbelong relation. Therefore a $t t$-soft semiregular space needs not be stable.

\section{Proposition 37.}

(i) A tt-soft semi-T $T_{i}$-space is always soft semi- $T_{i}$ for $i=$ $0,1,4$

(ii) A soft semiregular space is always tt-soft semiregular

(iii) A soft semi- $T_{3}$-space is always tt-soft semi- $T_{3}$.

Proof. Since $x \notin G_{P}$ implies $x \notin G_{P}$, then the proofs of (i) and (ii) follow.

To prove (iii), it suffices to prove that a soft semi- $T_{i}$-space is $t t$-soft semi- $T_{i}$ when $(X, \Omega, P)$ is soft semiregular. Suppose $x \neq y \in X$. Then, there exist two soft semiopen sets $U_{P}$ and $V_{P}$ so that $x \in U_{P}$ and $y \notin U_{P}$; and $y \in V_{P}$ and $x \notin V_{P}$. Since $U_{P}$ and $V_{P}$ are soft semiopen subsets of a soft semiregular space, then they are stable. So $y \notin U_{P}$ and $x \notin V_{P}$. Thus, $(X, \Omega, P)$ is $t t$-soft semi- $T_{1}$. Hence, we obtain the desired result.

The following examples clarifies that the converse of the above proposition is not always true.

Example 1. Let $P=\left\{p_{1}, p_{2}\right\}$. A family $\Omega=\left\{\tilde{\Phi}, \tilde{X}, G_{i_{P}}: i=1\right.$, $2, \cdots, 6\}$ is a soft topology on $X=\{v, w\}$, where

$$
\begin{aligned}
& G_{1_{P}}=\left\{\left(p_{1},\{v\}\right),\left(p_{2}, X\right)\right\} \\
& G_{2_{P}}=\left\{\left(p_{1}, X\right),\left(p_{2},\{w\}\right)\right\} \\
& G_{3_{P}}=\left\{\left(p_{1},\{v\}\right),\left(p_{2},\{w\}\right)\right\} \\
& G_{4_{P}}=\left\{\left(p_{1},\{w\}\right),\left(p_{2}, \varnothing\right)\right\} \\
& G_{5_{P}}=\left\{\left(p_{1}, \varnothing\right),\left(p_{2},\{v\}\right)\right\} \\
& G_{6_{P}}=\left\{\left(p_{1},\{w\}\right),\left(p_{2},\{v\}\right)\right\} .
\end{aligned}
$$

Since the soft sets $\left\{\left(p_{1},\{v\}\right),\left(p_{2},\{v\}\right)\right\}$ and $\left\{\left(p_{1},\{w\}\right)\right.$, $\left.\left(p_{2},\{w\}\right)\right\}$ are not soft semiopen subsets of $(X, \Omega, P)$, then it is not $t t$-soft semi- $T_{0}$. However, it is clear that $(X, \Omega, P)$ is a soft semi- $T_{1}$-space. Also, it is soft seminormal. Therefore, it is soft semi- $T_{4}$.

Example 2. Let $P=\left\{p_{1}, p_{2}\right\}$. A family $\Omega=\left\{\tilde{\Phi}, \tilde{X}, G_{i_{P}}: i=1\right.$, $2,3,4\}$ is a soft topology on $X=\{v, w\}$, where

$$
\begin{aligned}
& G_{1_{P}}=\left\{\left(p_{1},\{v\}\right),\left(p_{2},\{v\}\right)\right\} \\
& G_{2_{P}}=\left\{\left(p_{1},\{w\}\right),\left(p_{2},\{w\}\right)\right\} \\
& G_{3_{P}}=\left\{\left(p_{1},\{v\}\right),\left(p_{2}, X\right)\right\} \\
& G_{4_{P}}=\left\{\left(p_{1}, \varnothing\right),\left(p_{2},\{w\}\right)\right\} .
\end{aligned}
$$

It can be checked that a set in $(X, \Omega, P)$ is soft open iff it is soft semiopen. Since there exists an unstable soft semiopen set in $(X, \Omega, P)$, then $(X, \Omega, P)$ is not soft semiregular. Therefore, it is not soft semi- $T_{3}$. However, $(X, \Omega, P)$ is a $t t$-soft semiregular space. Also, it is a $t t$-soft semi- $T_{1}$-space. Hence, it is a $t t$-soft semi- $T_{3}$-space.

Before we show the relationship between $t$-soft semi$T_{i}$-spaces, we need to prove the next useful lemma.

Lemma 3. $(X, \Omega, P)$ is a tt-soft semi-T $T_{1}$-space iff $x_{P}$ is soft semiclosed for every $x \in X$.

Proof. Necessity: for any distinct point $y_{i} \in X$ than $x, \Omega$ contains a soft semiopen set and $G_{i_{p}}$ totally contains $y_{i}$ so that $x \notin G_{i_{p}}$. So $\bigcup_{i \in I} G_{i}(p)=X \backslash\{x\}$ and $x \notin \bigcup_{i \in I} G_{i}(p)$ for each $p \in P$. Thus, $\tilde{U}_{i \in I} G_{i_{P}}=X \widetilde{\backslash\{x\}}$ is soft semiopen. Hence, $x_{P}$ is soft semiclosed. 
Sufficiency: let $x \neq y$. By hypothesis, $x_{P}$ and $y_{P}$ are soft semiclosed sets. Then $x_{P}^{c}$ and $y_{P}^{c}$ are soft semiopen sets so that $x \in\left(y_{P}\right)^{c}$ and $y \in\left(x_{P}\right)^{c}$. Obviously, $y \notin\left(y_{P}\right)^{c}$ and $x \notin\left(x_{P}\right)^{c}$. Hence, $(X, \Omega, P)$ is $t t$-soft semi- $T_{1}$.

Proposition 38. Every $t$-soft semi- $T_{i}$-space is $t$-soft semi$T_{i-1}$ for $i=1,2,3,4$.

Proof. We suffice by proving the cases of $i=3,4$.

For $i=3$, let $x \neq y$ in a $t t$-soft semi- $T_{3}$-space $(X, \Omega, P)$. Then, $x_{P}$ is soft semiclosed. Since $y \notin x_{P}$ and $(X, \Omega, P)$ is tt-soft semiregular, then $\Omega$ contains two disjoint soft semiopen sets $G_{P}$ and $F_{P}$ satisfying $x_{P} \tilde{\subseteq} G_{P}$ and $y \in F_{P}$. Therefore, $(X, \Omega, P)$ is $t t$-soft semi- $T_{2}$.

For $i=4$, let $H_{P}$ be a soft semiclosed set so that $x \notin H_{P}$. Since $(X, \Omega, P)$ is $t t$-soft semi- $T_{1}$, then $x_{P}$ is soft semiclosed. Since $x_{P} \tilde{\cap} H_{P}=\tilde{\Phi}$ and $(X, \Omega, P)$ is soft seminormal, then there are disjoint soft semiopen sets $G_{P}$ and $F_{P}$ so that $H_{P} \subseteq G_{P}$ and $x_{P} \subseteq \sim F_{P}$. Hence, $(X, \Omega, P)$ is $t t$-soft semi- $T_{3}$.

We explain that the converse of proposition above is not always true by presenting the next example.

Example 1. Let $P=\left\{p_{1}, p_{2}\right\}$. A family $\Omega=\left\{\tilde{\Phi}, \tilde{X},\left\{\left(p_{1},\{x\}\right)\right.\right.$, $\left.\left(p_{2}, \varnothing\right)\right\}$ is a soft topology on $X=\{v, w\}$. The following soft sets with $\Omega$ are all soft semiopen subsets of $(X, \Omega, P)$.

$$
\begin{aligned}
& G_{1_{P}}=\left\{\left(p_{1},\{v\}\right),\left(p_{2},\{v\}\right)\right\} \\
& G_{2_{P}}=\left\{\left(p_{1},\{v\}\right),\left(p_{2},\{w\}\right)\right\} \\
& G_{3_{P}}=\left\{\left(p_{1},\{v\}\right),\left(p_{2}, X\right)\right\} \\
& G_{4_{P}}=\left\{\left(p_{1}, X\right),\left(p_{2},\{w\}\right)\right\} \\
& G_{5_{P}}=\left\{\left(p_{1}, X\right),\left(p_{2},\{w\}\right)\right\} .
\end{aligned}
$$

Since the soft sets $\left\{\left(p_{1},\{v\}\right),\left(p_{2},\{v\}\right)\right\}$ are a soft semiopen set in $(X, \Omega, P)$, then it is $t t$-soft semi- $T_{0}$. However, $\left\{\left(p_{1},\{w\}\right),\left(p_{2},\{w\}\right)\right\}$ is not a soft semiopen set in $(X, \Omega$, $P)$. Hence, it is not $t$-soft semi- $T_{1}$.

Example 2. Let $\Omega=\left\{\tilde{\Phi}, G_{P} \tilde{\subseteq} \mathbb{N}: G_{P}^{c}\right.$ is finite $\}$ be a soft topology on the set of natural numbers $\mathbb{N}$, where $P$ is a set of parameters. By a simple check, one notes that a class of soft semiopen and a class of soft open sets in $(\mathbb{N}, \Omega, P)$ coincide. For each $v \neq w \in \mathbb{N}$, we have $\mathbb{N} \widetilde{\backslash w}\}$ and $\widetilde{\mathbb{N} \backslash\{v\}}$ are soft semiopen sets so that $v \in \mathbb{N} \widetilde{\backslash w}\}$ and $w \notin \mathbb{N} \widetilde{\backslash w\}}$; and $w$ $\in \mathbb{N} \widetilde{\backslash}\{v\}$ and $v \notin \mathbb{N} \widetilde{\backslash v}\}$. Therefore, $(\mathbb{N}, \Omega, P)$ is tt-soft semi- $T_{1}$. In contrast, all nonnull proper semiopen sets have nonnull soft intersection. Hence, $(\mathbb{N}, \Omega, P)$ is not tt-soft semi- $T_{2}$.

Example 3. As we know, the coincidence of soft topological and classical topological spaces occurs if $E$ is a singleton. Then, it suffices to consider examples that satisfy a semi- $T_{2}$-space but not semi- $T_{3}$ and satisfy a semi- $T_{3}$-space but not semi- $T_{4}$.
In what follows, we found some properties of $t t$-soft semi- $T_{i}$ and $t t$-soft semiregular.

Lemma 39. Let $U_{P}$ be a subset of $(X, \Omega, P)$ and $x \in X$. Then $x \notin \bar{U}_{P}{ }^{s}$ iff there is a soft semiopen set $V_{P}$ totally containing $x$ so that $U_{P} \tilde{\cap} V_{P}=\tilde{\Phi}$.

Proof. Let $x \notin \bar{U}_{P}^{s}$. Then, $x \in\left(\bar{U}_{P}^{s}\right)^{c}=V_{P}$. So, $U_{P} \tilde{\cap} V_{P}=\tilde{\Phi}$. Conversely, if there exists a soft semiopen set $V_{P}$ totally containing $x$ so that $U_{P} \tilde{\cap} V_{P}=\tilde{\Phi}$, then $U_{P} \subseteq V_{P}^{c}$. Therefore, $\bar{U}_{P}{ }^{s} \subseteq V_{P}^{c}$. Since $x \notin V_{P}^{c}$, then $x \notin \bar{U}_{P}{ }^{s}$.

Proposition 40. If $(X, \Omega, P)$ is a tt-soft semi- $T_{0}$-space, then ${\overline{x_{P}}}^{s} \neq{\overline{y_{P}}}^{s}$ for every $x \neq y \in X$.

Proof. Let $x \neq y$ in a $t$-soft semi- $T_{0}$-space. Then, there is a soft semiopen set $U_{P}$ so that $x \in U_{P}$ and $y \notin U_{P}$ or $y \in U_{P}$ and $x \notin U_{P}$. Say, $x \in U_{P}$ and $y \notin U_{P}$. Now, $y_{P} \tilde{\cap} U_{P}=\tilde{\Phi}$. So, by the above lemma, $x \notin \overline{y_{P}}$. But $x \in \overline{x_{P}}$. Hence, we obtain the desired result.

Corollary 41. If $(X, \Omega, P)$ is a tt-soft semi- $T_{0}$-space, then $\bar{S}_{p}^{x^{s}} \neq{\overline{S^{y}}}_{p^{\prime}}$ for all $x \neq y$ and $p, p^{\prime} \in P$.

Theorem 42. Let $P$ be a finite set and $(X, \Omega, P)$ be soft hyperconnected. Then $(X, \Omega, P)$ is a tt-soft semi-T $T_{1}$-space if and only if $x_{P}=\tilde{\cap}\left\{U_{P}: x \in U_{P} \in \Omega^{s}\right\}$ for each $x \in X$.

Proof. To prove the "if" part, let $y \in X$. Then for each $x \in$ $X \backslash\{y\}$, we have a soft semiopen set $U_{P}$ so that $x \in U_{P}$ and $y \notin U_{P}$. Therefore $y \notin \cap \sim\left\{U_{P}: x_{P} \tilde{\subseteq} U_{P} \in \Omega^{s}\right\}$. Since $y$ is chosen arbitrary, then the desired result is proved.

To prove the "only if" part, let the given conditions be satisfied and let $x \neq y$. Let $|E|=m$. Since $y \notin x_{P}$, then for each $j=1,2, \cdots, m$ there is a soft semiopen set $U_{i_{P}}$ so that $y \notin U_{i}\left(e_{j}\right)$ and $x \in U_{i_{P}}$. Since $(X, \Omega, P)$ is soft hyperconnected, then it follows from Theorem 17 that $\tilde{\cap}_{i=1}^{i=m} U_{i_{P}}$ is a soft semiopen set so that $y \notin \tilde{\cap}_{i=1}^{i=m} U_{i_{P}}$ and $x \in \tilde{\cap}_{i=1}^{i=m} U_{i_{P}}$. Similarly, we can get a soft semiopen set $V_{P}$ so that $y \in V_{P}$ and $x \notin V_{P}$. Thus, $(X, \Omega, P)$ is a $t$-soft semi- $T_{1}$-space.

Theorem 43. If $(X, \Omega, P)$ is an extended tt-soft semi- $T_{1^{-}}$ space, then $S_{p}^{x}$ is soft semiclosed for all $S_{p}^{x} \in \tilde{X}$.

Proof. By Lemma 3, we have $X \widetilde{\backslash\{x\}}$ is a soft semiopen set. Since $(X, \Omega, P)$ is extended, then a soft set $H_{P}$, where $H(p)=\varnothing$ and $H\left(p^{\prime}\right)=X$ for each $p^{\prime} \neq p$, is a soft semiopen set. Therefore, $X \backslash\{x\}^{\sim} \cup^{\sim} H_{P}$ is soft semiopen. Thus, $\left(X \backslash\{x\}^{\sim} \cup^{\sim} H_{P}\right)^{c}=S_{p}^{x}$ is soft semiclosed.

Corollary 44. If $(X, \Omega, P)$ is an extended tt-soft semi- $T_{1^{-}}$ space, then the intersection of all soft semiopen sets containing $U_{P}$ is exactly $U_{P}$ for each $U_{P} \tilde{\subseteq} \tilde{X}$. 
Proof. Let $U_{P}$ be a soft subset of $\tilde{X}$. Since $S_{p}^{x}$ is a soft semiclosed set for every $S_{p}^{x} \in U_{P}^{c}$, then $\tilde{X} \backslash S_{p}^{x}$ is a soft semiopen set containing $U_{P}$. Therefore, $U_{P}=\tilde{\cap}\left\{\tilde{X} S_{p}^{x}: S_{p}^{x} \in U_{P}^{c}\right\}$, as required.

Theorem 45. Let $(X, \Omega, P)$ be finite soft hyperconnected. Then, $(X, \Omega, P)$ is tt-soft semi- $T_{2}$ if and only if it is $t$-soft semi- $T_{1}$.

Proof. Necessity: it is obtained from Proposition 38.

Sufficiency: for each $x \neq y$, we have $x_{P}$ and $y_{P}$ are soft semiclosed sets. Since $X$ is finite, then $\tilde{U}_{y \in X \backslash\{x\}} y_{P}$ and $\tilde{U}_{x \in X \backslash\{y\}} x_{P}$ are soft semiclosed sets. Since $(X, \Omega, P)$ is soft hyperconnected, then $\left(\mathrm{U}_{y \in X \backslash\{x\}}^{\sim} y_{P}\right)^{c}=x_{P}$ and $\left(\tilde{U}_{x \in X \backslash\{y\}} x_{P}\right)^{c}$ $=y_{P}$ are soft semiopen sets. The disjointness of $x_{P}$ and $y_{P}$ ends the proof that $(X, \Omega, P)$ is $t t$-soft semi- $T_{2}$.

Remark 46. In Example2, note that $x_{P}$ is not a soft semiopen set for each $x \in \mathbb{N}$. This clarifies that a soft set $x_{P}$ in a $t$-soft semi- $T_{1}$-space need not be soft semiopen if the universal set is infinite.

Theorem 47. $(X, \Omega, P)$ is tt-soft semiregular iff for every soft semiopen subset $F_{P}$ of $(X, \Omega, P)$ totally containing $x$, there is a soft semiopen set $V_{P}$ so that $x \in V_{P} \tilde{\subseteq} \bar{V}_{P}^{s} \tilde{\subseteq} F_{P}$.

Proof. Let $F_{P}$ be a soft semiopen set totally containing $x$. Then $F_{P}^{c}$ is semisoft closed and $x_{P} \tilde{\cap} F_{P}^{c}=\tilde{\Phi}$. So $\Omega$ contains two disjoint soft semiopen sets $U_{P}$ and $V_{P}$ satisfying $F_{P}^{c} \tilde{\subseteq} U_{E}$ and $x \in V_{P}$. Thus, $V_{P} \tilde{\subseteq} U_{P}^{c} \tilde{\subseteq} F_{P}$. Hence, $\bar{V}_{P}{ }^{s} \simeq U_{P}^{c} \tilde{\simeq} F_{P}$. Conversely, let $F_{P}^{c}$ be a soft semiclosed set. Then, for each $x \notin F_{P}^{c}$, we have $x \in F_{P}$. By hypothesis, there is a soft semiopen set $V_{P}$ totally containing $x$ so that $\bar{V}_{P}{ }^{s} \subseteq F_{P}$. Therefore, $F_{P}^{c}$ $\tilde{\subseteq}\left(\bar{V}_{P}{ }^{s}\right)^{c}$ and $V_{P} \tilde{\cap}\left(\bar{V}_{P}{ }^{s}\right)^{c}=\tilde{\Phi}$. Thus, $(X, \Omega, P)$ is tt-soft semiregular, as required.

Theorem 48. The three concepts below are identical if $(X$, $\Omega, P)$ is a tt-soft semiregular space.

(i) a tt-soft semi- $T_{2}$-space

(ii) a tt-soft semi- $T_{1}$-space

(iii) a tt-soft semi- $T_{0}$-space.

Proof. The directions $(i) \longrightarrow($ ii $)$ and $(i i) \longrightarrow($ iii $)$ are obvious.

To prove $($ iii $) \longrightarrow(i)$, let $x \neq y$ in a $t t$-soft semi- $T_{0}$-space $(X, \Omega, P)$. Then, there exists a soft semiopen set $G_{P}$ so that $x \in G_{P}$ and $y \notin G_{P}$, or $y \in G_{P}$ and $x \notin G_{P}$. Say, $x \in G_{P}$ and $y \notin$ $G_{P}$. Obviously, $x \notin G_{P}^{c}$ and $y \in G_{P}^{c}$. Since $(X, \Omega, P)$ is $t t$-soft semiregular, then there exist two disjoint soft semiopen sets $U_{P}$ and $V_{P}$ so that $x \in U_{P}$ and $y \in G_{P}^{c} \tilde{\subseteq} V_{P}$. Hence, $(X, \Omega$, $P)$ is $t t$-soft semi- $T_{2}$.
Proposition 49. Let $(X, \Omega, P)$ be finite soft hyperconnected. If $(X, \Omega, P)$ is a tt-soft semi-T $T_{2}$-space, then it $i$ tt-soft semiregular.

Proof. Let $H_{P}$ be a soft semiclosed set and $x \in X$ so that $x \notin H_{P}$. Then $x \neq y$ for each $y \Subset H_{P}$. By hypothesis, there are two disjoint soft semiopen sets $U_{i_{P}}$ and $V_{i_{p}}$ so that $x \in U_{i_{P}}$ and $y \in$ $V_{i_{p}}$. Since $\{y: y \in X\}$ is a finite set, then there is a finite number of soft semiopen sets $V_{i_{P}}$ so that $H_{P} \tilde{\simeq} \tilde{U}_{i=1}^{i=m} V_{i_{P}}$. Since $(X, \Omega, P)$ is soft hyperconnected, then it follows from Theorem 17 that $\tilde{\cap}_{i=1}^{i=m} U_{i_{p}}$ is a soft semiopen set containing $x$. Since $\left[\tilde{\cup}_{i=1}^{i=m} V_{i_{p}}\right] \tilde{\cap}\left[\tilde{\cap}_{i=1}^{i=m} U_{i_{p}}\right]=\tilde{\Phi}$, then $(X, \Omega, P)$ is $t t$-soft semiregular.

Corollary 50. The following concepts are identical if $(X, \Omega, P)$ is finite soft hyperconnected.

(i) a tt-soft semi- $T_{3}$-space

(ii) a tt-soft semi- $T_{2}$-space

(iii) a tt-soft semi-T $T_{1}$-space.

Proof. We obtain the directions $(i) \longrightarrow(i i)$ and $(i i) \longrightarrow(i i i)$ from Proposition 38.

We obtain the direction $($ iii $) \longrightarrow$ (ii) from Theorem 45 . We obtain the direction $(i i) \longrightarrow($ i $)$ from Proposition 49 .

Theorem 51. The property of being a tt-soft semi- $T_{i}$-space $(i=0,1,2,3)$ is a soft open hereditary.

Proof. We suffice by proving the cases of $i=3$.

Let $\left(Y, \Omega_{Y}, P\right)$ be a soft open subspace of a $t t$-soft semi$T_{3}$-space $(X, \Omega, P)$. To prove that $\left(Y, \Omega_{Y}, P\right)$ is $t t$-soft semi$T_{1}$, let $x \neq y \in Y$. Since $(X, \Omega, P)$ is a $t t$-soft semi- $T_{1}$-space, then there exist two soft semiopen sets $G_{P}$ and $F_{P}$ so that $x \in G_{P}$ and $y \notin G_{P}$; and $y \in F_{P}$ and $x \notin F_{P}$. Therefore, $x \in$ $U_{P}=\tilde{Y} \tilde{\cap} G_{P}$ and $y \in V_{P}=\tilde{Y} \tilde{\cap} F_{P}$ so that $y \notin U_{P}$ and $x \notin$ $V_{P}$. It follows from Proposition 18 that $U_{P}$ and $V_{P}$ are soft semiopen subsets of $\left(Y, \Omega_{Y}, P\right)$, so that $\left(Y, \Omega_{Y}, P\right)$ is $t t$-soft semi- $T_{1}$.

To prove that $\left(Y, \Omega_{Y}, P\right)$ is $t t$-soft semiregular, let $y \in Y$ and $F_{P}$ be a soft semiclosed subset of $\left(Y, \Omega_{Y}, P\right)$ so that $y \notin$ $F_{P}$. Then, $F_{P} \tilde{U} \tilde{Y}^{c}$ is a soft semiclosed subset of $(X, \Omega, P)$ so that $y \notin F_{P} \tilde{U} \widetilde{Y}^{c}$. Therefore, there exist disjoint soft semiopen subsets $U_{P}$ and $V_{P}$ of $(X, \Omega, P)$ so that $F_{P} \tilde{U} \tilde{Y}^{c} \tilde{\subseteq} U_{P}$ and $y \in$ $V_{P}$. Now, $U_{P} \tilde{\cap} \tilde{Y}$ and $V_{P} \tilde{\cap} \tilde{Y}$ are disjoint soft semiopen subsets of $\left(Y, \Omega_{Y}, P\right)$ so that $F_{P} \tilde{\subseteq} U_{P} \tilde{\cap} \tilde{Y}$ and $y \in V_{P} \tilde{\cap} \tilde{Y}$. Thus, $\left(Y, \Omega_{Y}, P\right)$ is $t$-soft semiregular.

Hence, $\left(Y, \Omega_{Y}, P\right)$ is $t t$-soft semi- $T_{3}$, as required.

Theorem 52. Let $(X, \Omega, P)$ be extended and $i=0,1,2,3,4$. Then, $(X, \Omega, P)$ is tt-soft semi-T $T_{i}$ iff $\left(X, \Omega_{p}\right)$ is semi- $T_{i}$ for each $p \in P$. 
Proof. We prove the theorem in the case of $i=4$, and one can similarly prove the other cases.

Necessity: let $x \neq y$ in $X$. Then, there exist two soft semiopen sets $U_{P}$ and $V_{P}$ so that $x \in U_{P}$ and $y \notin U_{P}$; and $y \in V_{P}$ and $x \notin V_{P}$. Obviously, $x \in U(p)$ and $y \notin U(p)$; and $y \in V(p)$ and $x$ $\notin V(p)$. Since $(X, \Omega, P)$ is extended, then it follows from Theorem 26 that $U(p)$ and $V(p)$ are semiopen subsets of $\left(X, \Omega_{p}\right)$ for each $p \in P$. Thus, $\left(X, \Omega_{p}\right)$ is a semi- $T_{1}$-space. To prove that $\left(X, \Omega_{p}\right)$ is seminormal, let $F_{p}$ and $H_{p}$ be two disjoint semiclosed subsets of $\left(X, \Omega_{p}\right)$. Let $F_{P}$ and $H_{P}$ be two soft sets given by $F(p)=F_{p}, H(p)=H_{p}$, and $F\left(p^{\prime}\right)=H\left(p^{\prime}\right)=\varnothing$ for each $p^{\prime} \neq p$. It follows from Theorem 26 that $F_{P}$ and $H_{P}$ are two disjoint soft semiclosed subsets of $(X, \Omega, P)$. By hypothesis, there exist two disjoint soft semiopen sets $G_{P}$

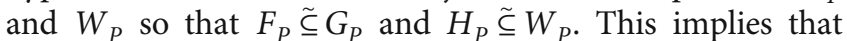
$F(p)=F_{p} \subseteq G(p)$ and $H(p)=H_{p} \subseteq W(p)$. Since $(X, \Omega, P)$ is extended, then it follows from Theorem 26 that $G(p)$ and $W(p)$ are semiopen subsets of $\left(X, \Omega_{p}\right)$. Thus, $\left(X, \Omega_{p}\right)$ is a seminormal space. Hence, it is a semi- $T_{4}$-space.

Sufficiency: let $x \neq y$ in $X$. Then there exists two semiopen subsets $U_{p}$ and $V_{p}$ of $\left(X, \Omega_{p}\right)$ so that $x \in U_{p}$ and $y \notin U_{p}$; and $y \in V_{p}$ and $x \notin V_{p}$. Let $U_{P}$ and $V_{P}$ be two soft sets given by $U(p)=U_{p}$ and $V(p)=V_{p}$ for each $p \in P$. Since $(X, \Omega, P)$ is extended, then it follows from Theorem 26 that $U_{P}$ and $V_{P}$ are soft semiopen subsets of $(X, \Omega, P)$ so that $x \in U_{P}$ and $y$ $\notin U_{P}$; and $y \in V_{P}$ and $x \notin V_{P}$. Thus, $(X, \Omega, P)$ is a $t$-soft semi- $T_{1}$-space. To prove that $(X, \Omega, P)$ is soft seminormal, let $F_{P}$ and $H_{P}$ be two disjoint soft semiclosed subsets of $(X$, $\Omega, P)$. Since $(X, \Omega, P)$ is extended, then it follows from Theorem 26 that $F(p)$ and $H(p)$ are two disjoint semiclosed subsets of $\left(X, \Omega_{p}\right)$. By hypothesis, there exist two disjoint semiopen subsets $G_{p}$ and $W_{p}$ of $\left(X, \Omega_{p}\right)$ so that $F(p) \subseteq G_{p}$ and $H(p) \subseteq W_{p}$. Let $G_{P}$ and $W_{P}$ be two soft sets given by $G$ $(p)=G_{p}$ and $W(p)=W_{p}$ for each $p \in P$. Since $(X, \Omega, P)$ is extended, then it follows from Theorem 26 that $G_{P}$ and $W_{P}$ are two disjoint soft semiopen subsets of $(X, \Omega, P)$ so that $F_{P} \tilde{\simeq} G_{P}$ and $H_{P} \tilde{\subseteq} W_{P}$. Thus, $(X, \Omega, P)$ is soft seminormal. Hence, it is a $t$-soft semi- $T_{4}$-space.

In the following examples, we show that a condition of an extended soft topology given in the above theorem is not superfluous.

Example 1. Let $(X, \Omega, P)$ be the same as in Example1. We showed that $(X, \Omega, P)$ is not tt-soft semi-T $T_{0}$. On the other hand, $\Omega_{p_{1}}$ and $\Omega_{p_{2}}$ are the discrete topology on $X$. Hence, the two parametric topological spaces $\left(X, \Omega_{p_{1}}\right)$ and $\left(X, \Omega_{p_{2}}\right)$ are semi- $T_{4}$.

Theorem 53. The property of being a tt-soft semi- $T_{i}$-space $(i=0,1,2)$ is preserved under a finite product soft spaces.

Proof. We prove the theorem in case of $i=2$. The other cases follow similar lines.

Let $\left(\mathrm{X}_{1}, \Omega_{1}, E_{1}\right)$ and $\left(X_{2}, \Omega_{2}, E_{2}\right)$ be two $t t$-soft semi- $T_{2}$ spaces and let $\left(x_{1}, y_{1}\right) \neq\left(x_{2}, y_{2}\right)$ in $X_{1} \times X_{2}$. Then, $x_{1} \neq x_{2}$ or $y_{1} \neq y_{2}$. Without loss of generality, let $x_{1} \neq x_{2}$. Then there exist two disjoint soft semiopen subsets $G_{E_{1}}$ and $H_{E_{1}}$ of $\left(X_{1}, \Omega_{1}, E_{1}\right)$ so that $x_{1} \in G_{E_{1}}$ and $x_{2} \notin G_{E_{1}}$; and $x_{2} \in H_{E_{1}}$ and $x_{1} \notin H_{E_{1}}$. Obviously, $G_{E_{1}} \times \widetilde{X_{2}}$ and $H_{E_{1}} \times \widetilde{X_{2}}$ are two disjoint soft semiopen subsets $X_{1} \times X_{2}$ so that $\left(x_{1}, y_{1}\right) \in G_{E_{1}} \times \widetilde{X_{2}}$ and $\left(x_{2}, y_{2}\right) \notin G_{E_{1}} \times \widetilde{X_{2}}$; and $\left(x_{2}, y_{2}\right) \in H_{E_{1}} \times \widetilde{X_{2}}$ and $\left(x_{1}, y_{1}\right) \notin$ $H_{E_{1}} \times \widetilde{X_{2}}$. Hence, $X_{1} \times X_{2}$ is a $t t$-soft semi- $T_{2}$-space. result.

For the sake of brevity, we omit the proof of the following

Theorem 54. The property of being a tt-soft semi- $T_{i}$-space is an additive property for $i=0,1,2,3,4$.

We complete this section by discussing some interrelations between $t t$-soft semi- $T_{i}$-spaces $(i=2,3,4)$ and soft semicompact spaces.

Proposition 55. A stable soft semicompact set in a tt-soft semi-T $T_{2}$-space is soft semiclosed.

Proof. It follows from Proposition 22 and Remark 35.

Theorem 56. Let $H_{p}$ be a soft semicompact subset of a soft hyperconnected $t t$-soft semi-T $T_{2}$-space. If $x \notin H_{P}$, then there are disjoint soft semiopen sets $U_{P}$ and $V_{P}$ so that $x \in U_{P}$ and $H_{P} \subseteq V_{P}$.

Proof. Let $x \notin H_{P}$. Then $x \neq y$ for each $y \Subset H_{P}$. Since $(X, \Omega, P)$ is a $t t$-soft semi- $T_{2}$-space, then there exist disjoint soft semiopen sets $U_{i_{p}}$ and $V_{i_{p}}$ so that $x \in U_{i_{p}}$ and $y \in V_{i_{p}}$. Therefore, $\left\{V_{i_{P}}\right\}$ forms a soft semiopen cover of $H_{P}$. Since $H_{P}$ is soft semicompact, then $H_{P} \subseteq \mathrm{U}_{\substack{i=1 \\ i=1}}^{\sim} V_{i_{p}}$. By the soft hyperconnectedness of $(X, \Omega, P)$, we obtain $\widetilde{\bigcap}_{i=1}^{i=n} U_{i_{p}}=U_{P}$ is a soft semiopen set. Hence, we obtain the desired result.

Theorem 57. Every soft hyperconnected, soft semicompact, and $t$-soft semi- $T_{2}$-space is $t$-soft semiregular.

Proof. Let $H_{P}$ be a soft semiclosed subset of soft semicompact and $t t$-soft semi- $T_{2}$-space $(X, \Omega, P)$ so that $x \notin H_{P}$. Then, $H_{P}$ is soft semicompact. By Theorem 56, there exist disjoint soft semiopen sets $U_{P}$ and $V_{P}$ so that $x \in U_{P}$ and $H_{P} \subseteq V_{P}$. Thus, $(X, \Omega, P)$ is $t t$-soft semiregular.

Corollary 58. Every soft hyperconnected, soft semicompact, and $t$-soft semi-T $T_{2}$-space is $t$-soft semi- $T_{3}$.

Lemma 59. Let $F_{P}$ be a soft semiopen subset of a soft semiregular space. Then, for each $S_{p}^{x} \in F_{P}$, there exists a soft semiopen set $G_{P}$ so that $S_{p}^{x} \in \bar{G}_{P}{ }^{s} \subseteq F_{P}$.

Proof. Let $F_{P}$ be a soft semiopen set so that $S_{p}^{x} \in F_{P}$. Then, $x \notin F_{P}^{c}$. Since $(X, \Omega, P)$ is soft semiregular, then there exist two disjoint soft semiopen sets $G_{P}$ and $W_{P}$ totally containing 
$x$ and $F_{P}^{c}$, respectively. Thus, $x \in G_{P} \tilde{\subseteq} W_{P}^{c} \tilde{\subseteq} F_{P}$. Hence, $S_{p}^{x} \in G_{P} \tilde{\simeq} \bar{G}_{P}{ }^{s} \tilde{\subseteq} W_{P}^{c} \tilde{\subseteq} F_{P}$.

Theorem 60. Let $H_{P}$ be a soft semicompact subset of a soft semiregular space and $F_{P}$ be a soft semiopen set containin $H_{P}$. Then, there exists a soft semiopen set $G_{P}$ so that $H_{P}$ $\tilde{\subseteq} G_{P} \tilde{\subseteq} \bar{G}_{P}{ }^{s} \tilde{\subseteq} F_{P}$.

Proof. Let the given conditions be satisfied. Then, for each $S_{p}^{x} \in H_{P}$, we have $S_{p}^{x} \in F_{P}$. Therefore, there is a soft semiopen set $W_{x e_{p}}$ so that $S_{p}^{x} \in W_{x e_{p}} \tilde{\subseteq} W_{x e_{p}}{ }^{s} \tilde{\subseteq} F_{P}$. Now, $\left\{W_{x e_{p}}: S_{p}^{x} \epsilon\right.$ $\left.F_{P}\right\}$ is a soft semiopen cover of $H_{P}$. Since $H_{P}$ is soft semicompact, then $H_{P} \tilde{\subseteq} \tilde{U}_{i=1}^{i=n} W_{x e_{P}}$. Putting $G_{P}=\tilde{\cup}_{i=1}^{i=n} W_{x e_{p}}$. Thus, $H_{P} \tilde{\simeq} G_{P} \tilde{\subseteq} \bar{G}_{P}{ }^{s} \tilde{\subseteq} F_{P}$.

Corollary 61. If $(X, \Omega, P)$ is soft semicompact and soft semi$T_{3}$, then it is tt-soft semi- $T_{4}$.

Proof. Suppose that $F_{1_{P}}$ and $F_{2_{P}}$ are two disjoint soft semiclosed sets. Then, $F_{2_{P}} \tilde{\simeq} F_{1_{p}}^{c}$. Since $(X, \Omega, P)$ is soft semicompact, then $F_{2_{P}}$ is soft semicompact and since $(X, \Omega, P)$ is soft semiregular, then there is a soft semiopen set $G_{P}$ so that $F_{2_{P}} \tilde{\subseteq} G_{P} \tilde{\simeq} \bar{G}_{P}{ }^{s} \tilde{\subseteq} F_{1_{P}}^{c}$. Obviously, $F_{2_{P}} \tilde{\subseteq} G_{P}, F_{1_{P}} \tilde{\subseteq}\left(\bar{G}_{P}{ }^{s}\right)^{c}$ and $G_{P} \tilde{\cap}\left(\bar{G}_{P}{ }^{s}\right)^{c}=\tilde{\Phi}$. Thus, $(X, \Omega, P)$ is soft seminormal. Since $(X, \Omega, P)$ is soft semi- $T_{3}$, then it is $t t$-soft semi- $T_{1}$. Hence, it is $t t$-soft semi- $T_{4}$.

\section{Semifixed Soft Points of Soft Mappings}

In this section, we introduce a semifixed soft point property and investigate some main features, in particular, those related to parametric topological spaces.

Theorem 62. Let $\left\{\mathscr{B}_{n}: n \in \mathbb{N}\right\}$ be a collection of soft subsets of a soft semicompact space $(X, \Omega, P)$ satisfying the following:

(i) $\mathscr{B}_{n} \neq \tilde{\Phi}$ for each $n \in \mathbb{N}$

(ii) $\mathscr{B}_{n}$ is a soft semiclosed set for each $n \in \mathbb{N}$

(iii) $\mathscr{B}_{n+1} \simeq \mathscr{B}_{n}$ for each $n \in \mathbb{N}$.

Then, $\tilde{\cap}_{n \in \mathbb{N}} \mathscr{B}_{n} \neq \tilde{\Phi}$.

Proof. Suppose that $\tilde{\cap}_{n \in \mathbb{N}} \mathscr{B}_{n}=\tilde{\Phi}$. Then, $\tilde{\cup}_{n \in \mathbb{N}} \mathscr{B}_{n}^{c}=\tilde{X}$. It follows from (ii) that $\left\{\mathscr{B}_{n}^{c}: n \in \mathbb{N}\right\}$ is a soft semiopen cover of $\tilde{X}$. By hypothesis of soft semicompactness, there exist $i_{1}$, $i_{2}, \cdots, i_{k} \in \mathbb{N}, \quad i_{1}<i_{2}<\cdots<i_{k}$ so that $\tilde{X}=\mathscr{B}_{i_{1}}^{c} \tilde{\cup} \mathscr{B}_{i_{2}}^{c} \tilde{\cup} \cdots$ $\tilde{\cup} \mathscr{B}_{i_{k}}^{c}$. It follows from (iii) that $\mathscr{B}_{i_{k}} \tilde{\subseteq} \tilde{X}=\mathscr{B}_{i_{1}}^{c} \tilde{\cup} \mathscr{B}_{i_{2}}^{c} \tilde{\cup} \cdots$ $\tilde{\cup} \mathscr{B}_{i_{k}}^{c}=\left[\mathscr{B}_{i_{1}} \tilde{\cap} \mathscr{B}_{i_{2}} \tilde{\cap} \cdots \tilde{\cap} \mathscr{B}_{i_{k}}\right]^{c}=\mathscr{B}_{i_{k}}^{c}$. This yields a contradiction. Thus, we obtain the proof that $\tilde{\cap}_{n \in \mathbb{N}} \mathscr{B}_{n} \neq \tilde{\Phi}$.

Proposition 63. Let $(X, \Omega, P)$ be a soft semicompact and soft semi- $T_{2}{ }_{2}$-space and $g_{\varphi}:(X, \Omega, P) \longrightarrow(X, \Omega, P)$ be a soft semi *-continuous map. Then there exists a unique soft point $S_{p}^{x} \in \tilde{X}$ of $g_{\varphi}$.

Proof. Let $\quad\left\{\mathscr{B}_{1}=g_{\varphi}(\tilde{X})\right.$ and $\mathscr{B}_{n}=g_{\varphi}\left(\mathscr{B}_{n-1}\right)=g_{\varphi}^{n}(\tilde{X})$ for each $n \in \mathbb{N}\}$ be a family of soft subsets of $(X, \Omega, P)$. It is clear that $\mathscr{B}_{n+1} \tilde{\subseteq} \mathscr{B}_{n}$ for each $n \in \mathbb{N}$. Since $g_{\varphi}$ is soft semi ${ }^{*}$-continuous, then $\mathscr{B}_{n}$ is a soft semicompact set for each $n \in \mathbb{N}$ and since $(X, \Omega, P)$ is soft semi- $T_{2}^{\prime}$, then $\mathscr{B}_{n}$ is also a soft semiclosed set for each $n \in \mathbb{N}$. It follows from Theorem 62 that $(H, P)=\tilde{\cap}_{n \in \mathbb{N}} \mathscr{B}_{n}$ is a nonnull soft set. Note that $g_{\varphi}(H, P)=g_{\varphi}\left(\tilde{n}_{n \in \mathbb{N}} g_{\varphi}^{n}(\tilde{X})\right) \tilde{\subseteq} \tilde{\cap}_{n \in \mathbb{N}} g_{\varphi}^{n+1}(\tilde{X}) \tilde{\subseteq} \tilde{\cap}_{n \in \mathbb{N}} g_{\varphi}^{n}(\tilde{X})$ $=(H, P)$. To show that $(H, P) \tilde{\subseteq} g_{\varphi}(H, P)$, suppose that there is a $S_{p}^{x} \in(H, P)$ so that $S_{p}^{x} \notin \mathscr{g}_{\varphi}(H, P)$. Let $\mathscr{C}_{n}=g_{\varphi}^{-1}$ $\left(S_{p}^{x}\right) \tilde{\cap} \mathscr{B}_{n}$. Obviously, $\mathscr{C}_{n} \neq \tilde{\Phi}$ and $\mathscr{C}_{n} \tilde{\simeq} \mathscr{C}_{n-1}$ for each $n \in \mathbb{N}$. Now, $g_{\varphi}^{-1}\left(S_{p}^{x}\right)$ is a soft compact subset of a soft semi- $T^{\prime}{ }_{2}$-space, then $g_{\varphi}^{-1}\left(S_{p}^{x}\right)$ is soft closed; therefore, $\mathscr{C}_{n}$ is a soft semiclosed set for each $n \in \mathbb{N}$. By Theorem 62, there exists a soft point $P_{m}^{y}$ so that $P_{m}^{y} \in g_{\varphi}^{-1}\left(S_{p}^{x}\right) \tilde{\cap} \mathscr{B}_{n}$. Therefore, $S_{p}^{x}=g_{\varphi}\left(P_{m}^{y}\right) \in g_{\varphi}(H, P)$. This is a contradiction. Thus, $g_{\varphi}(H, P)=(H, P)$. Hence, the proof is complete.

Definition 64.

(i) $(X, \Omega, P)$ is said to have a semifixed soft point property if every soft semi ${ }^{*}$-continuous map $g_{\varphi}$ : $(X, \Omega, P) \longrightarrow(X, \Omega, P)$ has a fixed soft point

(ii) A property is said to be a semi* ${ }^{*}$-soft topological property if the property is preserved by soft semi ${ }^{*}$ homeomorphism maps.

Proposition 65. The property of being a semifixed soft point is a semi*-soft topological property.

Proof. Let $(X, \Omega, P)$ and $(Y, \theta, P)$ be a soft semi*-homeomorphic. Then, there is a bijective soft map $f_{\varphi}:(X, \Omega, P)$ $\longrightarrow(Y, \theta, P)$ so that $f_{\varphi}$ and $f_{\varphi}^{-1}$ are soft semi*-continuous. Since $(X, \Omega, P)$ has a semifixed soft point property, then every soft semi*-continuous map $g_{\varphi}:(X, \Omega, P) \longrightarrow(X, \Omega$, $P)$ has a semifixed soft point. Now, let $h_{\varphi}:(Y, \theta, P) \longrightarrow$ $(Y, \theta, P)$ be a soft semi*-continuous. Obviously, $h_{\varphi} \circ f_{\varphi}$ : $(X, \Omega, P) \longrightarrow(Y, \theta, P)$ is a soft semi*-continuous. Also, $f_{\varphi}^{-1} \circ h_{\varphi} \circ f_{\varphi}:(X, \Omega, P) \longrightarrow(X, \Omega, P)$ is a soft semi*-continuous. Since $(X, \Omega, P)$ has a semifixed soft point property, then $f_{\varphi}^{-1}\left(h_{\varphi}\left(f_{\varphi}\left(S_{p}^{x}\right)\right)\right)=S_{p}^{x}$ for some $S_{p}^{x} \in \tilde{X}$. consequently, $f_{\varphi}$ $\left(f_{\varphi}^{-1}\left(h_{\varphi}\left(f_{\varphi}\left(S_{p}^{x}\right)\right)\right)\right)=f_{\varphi}\left(S_{p}^{x}\right)$. This implies that $h_{\varphi}\left(f_{\varphi}\left(S_{p}^{x}\right)\right)=$ $f_{\varphi}\left(S_{p}^{x}\right)$. Thus, $f_{\varphi}\left(S_{p}^{x}\right)$ is a semifixed soft point of $h_{\varphi}$. Hence, $(Y, \theta, P)$ has a semifixed soft point property, as required.

Before we investigate a relationship between soft topological space and their parametric topological spaces in terms of possessing a fixed (soft) point, we need to prove the following result. 
Theorem 66. If $\Omega$ is extended, then $g_{\varphi}:(X, \Omega, P) \longrightarrow(Y, \theta$ $, P)$ is soft semi ${ }^{*}$-continuous iff $g:\left(X, \Omega_{p}\right) \longrightarrow\left(Y, \theta_{\phi(p)}\right)$ is semi ${ }^{*}$-continuous.

Proof. Necessity: let $U$ be a semiopen subset of $\left(Y, \theta_{\phi(p)}\right)$. Then $\theta$ contains a soft semiopen set $G_{P}$ satisfying $G(\phi(p))$ $=U$. Since $g_{\varphi}$ is a soft semi*-continuous map, then $g_{\phi}^{-1}\left(G_{P}\right)$ is a soft semiopen set. Definition 8 implies that a soft subset $g_{\phi}^{-1}\left(G_{P}\right)=\left(g_{\phi}^{-1}(G)\right)_{P}$ of $(X, \Omega, P)$ is given by $g_{\phi}^{-1}(G)(p)=$ $g^{-1}(G(\phi(p)))$ for each $p \in P$. By hypothesis, $\Omega$ is extended; we obtain from Theorem 26 that a subset $g^{-1}(G(\phi(p)))=$ $g^{-1}(U)$ of $\left(X, \Omega_{p}\right)$ is semiopen. Hence, a map $g$ is semi*continuous.

Sufficiency: let $G_{P}$ be a soft semiopen subset of $(Y, \theta, P)$. According to Definition 8 , a soft subset $g_{\phi}^{-1}\left(G_{P}\right)=$ $\left(g_{\phi}^{-1}(G)\right)_{P}$ of $(X, \Omega, P)$ is given by $g_{\phi}^{-1}(G)(p)=g^{-1}(G(\phi(p))$ ) for each $p \in P$. Since a map $g$ is semi*-continuous, then a subset $g^{-1}(G(\phi(p)))$ of $\left(X, \Omega_{p}\right)$ is semiopen. By hypothesis, $\Omega$ is extended; we obtain from Theorem 26 that $g_{\phi}^{-1}\left(G_{P}\right)$ is a soft semiopen subset of $(X, \Omega, P)$. Hence, a soft map $g_{\varphi}$ is soft semi $*$-continuous.

Definition 67. $(X, \Omega)$ is said to have a semifixed point property if every semi*-continuous map $g:(X, \Omega) \longrightarrow(X, \Omega)$ has a fixed point.

Proposition 68. $(X, \Omega, P)$ has the property of a semifixed soft point iff $\left(X, \Omega_{p}\right)$ has the property of a semifixed point for each $p \in P$.

Proof. Necessity: let $(X, \Omega, P)$ has the property of a semifixed soft point. Then every soft semi*-continuous map $g_{\varphi}:(X$, $\Omega, P) \longrightarrow(X, \Omega, P)$ has a fixed soft point. Say, $S_{p}^{x}$. It follows from the above theorem that $g_{p}:\left(X, \Omega_{p}\right) \longrightarrow\left(X, \theta_{\phi(p)}\right)$ is semi*-continuous. Since $S_{p}^{x}$ is a fixed soft point of $g_{\varphi}$, then it must be that $g_{p}(x)=x$. Thus, $g_{p}$ has a fixed point. Hence, we obtain the desired result.

Sufficiency: let $\left(X, \Omega_{p}\right)$ has the property of a semifixed point for each $p \in P$. Then, every semi*-continuous map $g_{p}:\left(X, \Omega_{p}\right) \longrightarrow\left(X, \theta_{\phi(p)}\right)$ has a fixed point. Say, $x$. It follows from the above theorem that $g_{\varphi}:(X, \Omega, P) \longrightarrow(X, \theta, P)$ is soft semi*-continuous. Since $x$ is a fixed point of $g_{p}$, then it must be that $g_{\varphi}\left(S_{p}^{x}\right)=S_{p}^{x}$. Thus, $g_{\varphi}$ has a fixed soft point. Hence, we obtain the desired result.

\section{Conclusion}

This work is a contribution of studying new classes to soft topology. First, we have introduced new soft separation axioms with respect to ordinary points by using total belong and total nonbelong relations. This way of definition helps us to generalize existing comparable properties via general topology and to remove a strict condition of the shape of soft open and closed subsets of soft semiregular spaces. In gen- eral, we have studied their main properties and showed the interrelations between them with help of interesting examples. Second, we have defined semifixed soft point and investigated its basic properties.

As future works, we shall study them on the contents of supra soft topological spaces, minimal soft topological spaces, and soft weak structures. Finally, we hope that the concepts initiated herein will find their applications in many fields soon.

\section{Data Availability}

No data were used to support this study.

\section{Conflicts of Interest}

The author declares that he has no competing interests.

\section{Acknowledgments}

We are highly grateful to the anonymous referees for their helpful comments and suggestions for improving the paper.

\section{References}

[1] D. Molodtsov, "Soft set theory-first results," Computers \& Mathematcs with Applications, vol. 37, pp. 19-31, 1999.

[2] P. K. Maji, R. Biswas, and R. Roy, "Soft set theory," Computers \& Mathematcs with Applications, vol. 45, no. 4-5, pp. 555-562, 2003.

[3] P. K. Maji, R. Biswas, and R. Roy, "An application of soft sets in a decision making problem," Computers \& Mathematcs with Applications, vol. 44, no. 8-9, pp. 1077-1083, 2002.

[4] M. I. Ali, F. Feng, X. Liu, W. K. Min, and M. Shabir, "On some new operations in soft set theory," Computers \& Mathematcs with Applications, vol. 57, no. 9, pp. 1547-1553, 2009.

[5] T. M. Al-shami and M. E. El-Shafei, "T-soft equality relation," Turkish Journal of Mathematics, vol. 44, no. 4, pp. 1427-1441, 2020.

[6] M. Shabir and M. Naz, "On soft topological spaces," Computers \& Mathematcs with Applications, vol. 61, no. 7, pp. 1786-1799, 2011.

[7] W. K. Min, "A note on soft topological spaces," Computers \& Mathematcs with Applications, vol. 62, pp. 3524-3528, 2011.

[8] A. Aygünoglu and H. Aygün, "Some notes on soft topological spaces," Neural Computing and Applications, vol. 21, no. S1, pp. 113-119, 2012.

[9] T. Hida, "A comprasion of two formulations of soft compactness," Annals of Fuzzy Mathematics and Informatics, vol. 8, no. 4, pp. 511-524, 2014.

[10] T. M. Al-shami, "Comments on "Soft mappings spaces"," Scientific World Journal, vol. 2019, article 6903809, pp. 1-2, 2019.

[11] T. M. Al-shami, "Comments on some results related to soft separation axioms," Afrika Matematika, vol. 31, no. 7-8, pp. 1105-1119, 2020.

[12] T. M. Al-shami, M. E. El-Shafei, and M. Abo-Elhamayel, "Almost soft compact and approximately soft Lindelöf spaces," Journal of Taibah University for Science, vol. 12, no. 5 , pp. $620-630,2018$. 
[13] A. Kharal and B. Ahmad, "Mappings on soft classes," New Mathematics and Natural Computation, vol. 7, no. 3, pp. 471-481, 2011.

[14] D. Wardowski, "On a soft mapping and its fixed points," Fixed Point Theory and Applications, vol. 2013, no. 1, 2013.

[15] P. Majumdar and S. K. Samanta, "On soft mappings," Computers \& Mathematcs with Applications, vol. 60, no. 9, pp. 2666-2672, 2010.

[16] M. E. El-Shafei, M. Abo-Elhamayel, and T. M. Al-shami, "Partial soft separation axioms and soft compact spaces," Univerzitet $u$ Nišu, vol. 32, no. 13, pp. 4755-4771, 2018.

[17] T. M. Al-shami and M. E. El-Shafei, "Partial belong relation on soft separation axioms and decision making problem: two birds with one stone," Soft Computing, vol. 24, no. 7, pp. 5377-5387, 2020.

[18] M. E. El-Shafei and T. M. Al-shami, "Applications of partial belong and total non-belong relations on soft separation axioms and decision-making problem," Computational and Applied Mathematics, vol. 39, no. 3, 2020.

[19] S. Das and S. K. Samanta, "Soft metric," Annals of Fuzzy Mathematics and Informatics, vol. 6, no. 1, pp. 77-94, 2013.

[20] S. Das and S. K. Samanta, "Soft real sets, soft real numbers and their properties," Journal of Fuzzy Mathematics, vol. 20, no. 3, pp. 551-576, 2012.

[21] M. Abbas, G. Murtaza, and S. Romaguera, "Soft contraction theorem," Journal of nonlinear and convex analysis, vol. 16, no. 3, pp. 423-435, 2015.

[22] M. Abbas, G. Murtaza, and S. Romaguera, "Remarks on fixed point theory in soft metric type spaces," Univerzitet $u$ Nišu, vol. 33, no. 17, pp. 5531-5541, 2019.

[23] S. Mohinta and T. K. Samanta, "Variant of soft compatible, weakly soft commuting maps and common fixed point theorem," International Journal of Mathematical Sciences \& Applications, vol. 2, no. 2, 2016.

[24] S. Mohinta and T. K. Samanta, "A comparison of various type of soft compatible maps and common fixed point theorem-II," International Journal on Cybernetics \& Informatics, vol. 4, no. 5, pp. 1-18, 2015.

[25] M. I. Yazar, Ç. Gunduz, and S. Bayramov, "Fixed point theorems of soft contractive mappings," Univerzitet $u$ Nišu, vol. 30, no. 2, pp. 269-279, 2016.

[26] F. Feng, H. Fujita, M. I. Ali, R. R. Yager, and X. Liu, “Another view on generalized intuitionistic fuzzy soft sets and related multiattribute decision making methods," IEEE Transactions on Fuzzy Systems, vol. 27, no. 3, pp. 474-488, 2019.

[27] F. Feng, Z. Xu, H. Fujita, and M. Liang, "Enhancing PROMETHEE method with intuitionistic fuzzy soft sets," International Journal of Intelligent Systems, vol. 35, no. 7, pp. 10711104, 2020.

[28] J. C. R. Alcantud, "Soft open bases and a novel construction of soft topologies from bases for topologies," Mathematics, vol. 8, no. 5 , p. $672,2020$.

[29] I. Zorlutuna and H. Çakir, "On continuity of soft mappings," Applied Mathematics \& Information Sciences, vol. 9, no. 1, pp. 403-409, 2015.

[30] S. Nazmul and S. K. Samanta, "Neighbourhood properties of soft topological spaces," Annals of Fuzzy Mathematics and Informatics, vol. 6, no. 1, pp. 1-15, 2013.

[31] T. M. Al-shami and L. D. R. Kocinac, "The equivalence between the enriched and extended soft topologies," Applied and Computational Mathematics, vol. 18, no. 2, pp. 149-162, 2019.

[32] T. M. Al-shami and L. D. R. Kocinac, "Nearly soft Menger spaces," Journal of Mathematics, vol. 2020, Article ID 3807418, 9 pages, 2020.

[33] F. Feng, Y. M. Li, B. Davvaz, and M. I. Ali, "Soft sets combined with fuzzy sets and rough sets: a tentative approach," Soft Computing, vol. 14, no. 9, pp. 899-911, 2010.

[34] K. V. Babitha and J. J. Suntil, "Soft set relations and functions," Computers \& Mathematcs with Applications, vol. 60, no. 7, pp. 1840-1849, 2010.

[35] B. Chen, "Soft semi-open sets and related properties in soft topological spaces," Applied Mathematics \& Information Sciences, vol. 7, no. 1, pp. 287-294, 2013.

[36] A. Kandil, O. A. E. Tantawy, S. A. El-Sheikh, and A. M. Abd El-latif, "Soft connectedness via soft ideals," Journal of New results in science, vol. 4, pp. 90-108, 2014.

[37] T. M. Al-shami, M. E. El-Shafei, and M. Abo-Elhamayel, "Seven generalized types of soft semi-compact spaces, Korean," The Korean Journal of Mathematics, vol. 27, no. 3, pp. 661-690, 2019.

[38] J. Mahanta and P. K. Das, "On soft topological space via semiopen and semi-closed soft sets," Kyungpook National University, vol. 54, pp. 221-236, 2014.

[39] T. M. Al-shami, L. D. R. Kocinac, and B. A. Asaad, "Sum of soft topological spaces," Mathematics, vol. 8, no. 6, p. 990, 2020. 